\title{
Educating an oocyte- the complex dialogue between mentor and mentee
}

\author{
David F. Albertini ${ }^{1}$
}

Published online: 19 August 2017

(C) Springer Science+Business Media, LLC 2017

As an audience of aspiring and established clinicians and scientists, we share in the spirit of acquiring and validating knowledge to deepen our understanding of the complexities of human and animal reproduction. While our professional purpose as reproductive medicine and biology specialists may range from that of practitioner, researcher, or educator, in the end, we have all arrived at this point as a result of the highest levels of education within whatever environment we have been schooled.

Reaching the level of expertise and scholarship necessary for career advancement is founded on the protracted interaction between us - the learners - and our mentors, commencing with nurturing parental influences. In many ways, this pathway is not unlike that pursued by the mammalian oocyte when after months to years of "waiting" within the primordial follicle, physical growth becomes aligned with an intercellular conversation within the ovarian follicle. The result is differentiation of the oocyte into a mature cell capable of undertaking one of the most remarkable events in reproduction - the conversion of a single cell into an organism capable of reproducing itself!

Research outcomes from the practice of human ARTs have delineated many of the educational milestones and obstacles intercalated along the pathway of oocyte differentiation. Although basic science has for decades recognized the link between the education of an oocyte and the performance of an embryo [1], incontestable evidence for this nexus is now at hand in the case of human reproduction.

David F. Albertini

eicjarg@gmail.com

1 The Center for Human Reproduction, New York, NY, USA
Only recently has it become patently clear that when carefully analyzed with respect to natural cycle single-embryo transfer outcomes, the fate of any resulting conceptus is essentially sealed by the intrinsic properties of the oocyte itself [2]. If you are a member of the oocentrist movement, then these findings come as little surprise. On the other hand, for the selection strategists of the embryocentric variety, some have been trying to raise our level of consciousness on this matter for nearly a decade.

Hence, the importance of an intraovarian education emphasizing the biological fact that rescuing eggs from follicles destined to undergo atresia does not compromise the transfer or implantability of ART-produced embryos, but few in fact can make a baby as originally alluded to by Patrizio and Sakkas [3]. While age and $\mathrm{COH}$ among other factors embedded within the conventional practice of ARTs make contributions to the aura of biological inefficiency, facing the fact that our technologies are no match for whatever is going on inside the follicle is disheartening and may be insurmountable at the end of the day [4].

If so, the time to eavesdrop into the conversation between oocyte and somatic elements of the follicle is here. Over the next few months, JARG will bring our readership to the forefront on this matter. While the historical excuse for poor oocyte quality has resulted in the logically biased perception of aging and aneuploidy, whether attribution is assigned to the meiotic or mitotic mandates is of less import than recognizing and accepting the many mechanistic frailties that McCoy has addressed in a recent review article, which I strongly recommend to all [5].

Headlining our series of papers this month is a provocative review by Kushnir and colleagues that serves to update our knowledge base on the pathophysiology of anti-Müllerian hormone, expanding the ways that AMH may provide novel treatment strategies for a number of disease states (Potential 
therapeutic applications of human anti-Müllerian hormone (AMH) analogues in reproductive medicine 10.1007/s10815017-0977). In addition, the Weiss laboratory demonstrates the relationship between sourcing and systemic delivery of AMH (Anti-Mullerian hormone concentrations in individual follicular fluids within one stimulated IVF cycle resemble blood serum values. 10.1007/s10815-017-0908), and Sacchi and collaborators provide an important mechanistic model system for studying the impact of AMH on granulosa cell transcriptional regulation (The anti-Müllerian hormone (AMH) induces forkhead box L2 (FOXL2) expression in primary culture of human granulosa cells in vitro 10.1007/ s10815-017-0980). Finally, the family of receptors for TGFb-related molecules is at the center of the communication landscape for many of the autocrine and paracrine effectors integrating oogenesis and folliculogenesis, a subject tackled by Ribeiro and colleagues in the context of aging in cumulus cells (Age-related expression of TGF beta family receptors in human cumulus oophorus cells 10.1007/s10815-017-0930).

Whether protracted use of hormonal contraceptives influences follicular dynamics and oocyte number is a question raised by Letourneau and colleagues (Long-term hormonal contraceptive use is associated with a reversible suppression of antral follicle count and a break from hormonal contraception may improve oocyte yield 10.1007/s10815-017-0981). Yields are one thing, but what is known about the education of oocytes with respect to achieving full growth, and accumulation of the dowry components that will ultimately drive embryogenesis? The fascinating results contributed by the laboratory of Shalom-Paz now suggest that obesity impacts the size of retrieved oocytes, which seems to be at least partially responsible for the poor treatment outcomes long known to affect this population (Obesity results with smaller oocyte in in vitro fertilization/intracytoplasmic sperm injection cycles - a prospective study 10.1007/s10815-017-0975).

Our issue this month also draws attention to the field of fertility preservation in the context of whether advances in technology warrant closer inspection of clinical utility beyond the experimental moniker, at least in certain instances (Medical and elective fertility preservation: impact of removal of the experimental label from oocyte cryopreservation 10.1007/s10815-0170968), even though some areas such as tissue cryopreservation are in need of further experimentation in order to optimize protocols for routine use (Complete protection against cryodamage of cryopreserved whole bovine and human ovaries using DMSO as a cryoprotectant. 10.1007/s10815-017-0963).

The road to an advanced degree for a developmentally competent human oocyte remains bewildering in complexity owing to the many cell types and forms of communication required. And yet, we and our patients have been hearing about breakthroughs in the propagation of oocytes from stem cells and the elaboration of artificial ovaries using bioengineering principles that mimic some of the professorial principles employed during follicle development in vivo - at least in mice [6].

We will hear more about cutting edge strategies and their hopeful translational extensions in upcoming issues of JARG. In the meantime, the task of educating an oocyte remains a daunting undertaking and reinforces more than ever that Mother Nature's inherent power of selection-her modus operandus - is exercised at many levels of human reproduction. Be it follicle, embryo, uterus, or brain, human ART maintains a narrow focus with little regard to the fact that the platform to propagation of our species rests firmly in the hands of the chosen oocyte! After all, as Atwood and Meethal [7] have recently elaborated upon, with human ARTs, we are mingling and meddling with an endocrine/ autocrine/paracrine orchestra and most certainly, as yet undiscovered means by which the reproductive capacity of an organism can be realized.

With this issue, we welcome Michael Bloom to our editorial board and would like to acknowledge the thoughtful and substantive contributions of Kutluk Oktay and Carlos Plancha to the JARG effort over these past 8 years.

\section{References}

1. Zuccotti M, Redi CA, Garagna S. Study an egg today to make an embryo tomorrow. Int J Dev Biol. 2012;56(10-12):761-4.

2. Silber SJ, Kato K, Aoyama N, Yabuuchi A, Skaletsky H, Fan Y, et al. Intrinsic fertility of human oocytes. Fertil Steril. 2017;107(5):1232-7.

3. Patrizio P, Sakkas D. From oocyte to baby: a clinical evaluation of the biological efficiency of in vitro fertilization. Fertil Steril. 2009;91(4):1061-6.

4. Martin JR, Bromer JG, Sakkas D, Patrizio P. Live babies born per oocyte retrieved in a subpopulation of oocyte donors with repetitive reproductive success. Fertil Steril. 2010;94(6):2064-8.

5. McCoy RC. Mosaicism in preimplantation human embryos: when chromosomal abnormalities are the norm. Trends Genet. 2017;33(7): 448-63.

6. Laronda MM, Rutz AL, Xiao S, Whelan KA, Duncan FE, Roth EW, et al. A bioprosthetic ovary created using 3D printed microporous scaffolds restores ovarian function in sterilized mice. Nat Commun. 2017;8:15261.

7. Atwood CS, Vadakkadath MS. The spatiotemporal hormonal orchestration of human folliculogenesis, early embryogenesis and blastocyst implantation. Mol Cell Endocrinol. 2016;430:33-48. 